\title{
FORUM
}

\section{ISSUES IN MEDICINE \\ Clinical \\ pharmacology \\ becomes a specialty in South Africa}

A Walubo, K Barnes, E Kwizera,

O Greeff, B Rosenkranz, G Maartens

A Walubo is at the Department of Pharmacology, University of the Free State, Bloemfontein; K Barnes and G Maartens at the Division of Clinical Pharmacology, Department of Medicine, University of Cape Town; E Kwizera at the Department of Pharmacology, Walter Sisulu University, Mthatha, Eastern Cape; O Greeff at the Department of Pharmacology, University of Pretoria; and B Rosenkranz at the Division of Pharmacology, Stellenbosch University.

Corresponding author: A Walubo (waluboa@ufs.ac.za)

South Africa recently became the first African country where clinical pharmacology has been approved as a specialty. This article outlines the need for clinical pharmacologists, their role in advancing public health, the potential benefits to the country, and recommendations for ensuring a healthy future for the discipline.

S Afr Med J 2013;103(3):150-151. DOI:10.7196/SAMJ.6639

Clinical pharmacology was recently approved as a specialty in South Africa $(\mathrm{SA})^{[1]}$ - the first country in Africa to do so. This milestone occurred concurrently with the World Health Organization (WHO)'s, publication of a position paper entitled 'Clinical pharmacology in health care, teaching and research' ${ }^{\text {'2] }}$ which explains the benefits of clinical pharmacologists to decision-makers. Clinical pharmacologists are medical practitioners trained in 'the scientific discipline that involves all aspects of the relationship between drugs and humans. ${ }^{[2]}$

In this article, the current members of the Council for the College of Clinical Pharmacologists of SA outline the need for clinical pharmacologists, their role in advancing public health, and the potential benefits to SA. We conclude with recommendations on how to ensure a healthy future for the discipline.

\section{The need for clinical pharmacologists}

There has been an enormous increase in the number of therapeutic products and formulations. ${ }^{[3]}$ Unfortunately, this expanding therapeutic armamentarium has been associated with an increased incidence of adverse drug reactions, drug interactions, drug resistance, drug overdose and medical errors. ${ }^{[4]}$ Because the increasing cost of new drugs is a major concern, insight into pharmaco-economic evaluation of new interventions is of critical importance. The population of patients with special dosing requirements is expanding - to mention but a few: those 
at the extremes of age, with co-morbid conditions, and those who are pregnant. There have been calls for increased clinical research in SA that is largely focused on the robust evaluation of therapeutic interventions, and addressing health needs of the local population. [5,6] There is an urgent need for skilled clinical pharmacologists to undertake training and research and offer their services to ensure rational use of drugs in humans.

\section{The core functions of a clinical pharmacologist \\ Drug policy formulation and implementation}

A major function of clinical pharmacologists is the promotion of the rational use of medicines. ${ }^{[2]}$ Clinical pharmacologists should be part of institutional, regional and national teams formulating drug policies, and thereafter participate in their implementation and continuous evaluation. The policy for rational use of medicines includes Standard Treatment Guidelines and Essential Medicines List, pharmacy therapeutics committees, prescribing policy, adverse drug reaction reporting/pharmaco-vigilance, education and training, continuing medical education, provision of information on medicines to healthcare workers, public/consumer education, and medicine promotion and advertising. ${ }^{[., 8]}$

\section{Clinical services}

Clinical pharmacologists serve as consultants in healthcare facilities as part of a multidisciplinary team including management personnel, clinicians, pharmacists, nurses etc. They are involved in, for example, consulting on complex therapeutic problems, drug use in special populations, therapeutic drug monitoring, information on drugs, diagnosis and management of overdose and poisoning, developing systems for reporting adverse drug reactions, and conducting therapeutic audits and drug utilisation reviews.

\section{Clinical research}

Clinical pharmacologists undertake clinical research related to all aspects of the science of therapeutics: the effectiveness and safety of registered drugs, drug utilisation, and the development of new drugs. As members of relevant committees (ethics or research or regulatory), clinical pharmacologists evaluate clinical research protocols to ensure the safety of research subjects and the validity of the proposed research.

\section{Education and training}

Clinical pharmacologists play a key role in the training of health professionals in the rational use of drugs at both under- and postgraduate levels.

In summary: Establishment of clinical pharmacology services ensures prioritisation of patient safety with regard to use of medicines; effective prevention and curative strategies; elimination of ambiguity in the utilisation of the services of a clinical pharmacologist in specific circumstances; setting minimum standards and therefore expectations for clinical pharmacology services; training and research; and career progression for those choosing to specialise as clinical pharmacologists.

\section{Ensuring a future for clinical pharmacology}

Clinical pharmacology is an extremely scarce skill in SA. Clinical pharmacologists with appropriate qualifications and experience are encouraged to apply to the HPCSA for registration as specialists under the 'grandfather clause' and to become affiliate members of the College of Clinical Pharmacologists of South Africa to contribute to the growth of the discipline in this country. Currently, five health science faculties have the capacity to train registrars in clinical pharmacology (Stellenbosch University, University of Cape Town, University of Limpopo, University of the Free State and University of Pretoria), but only one institution presently offers such training. There is an urgent need to expand training programmes and registrar posts for clinical pharmacology. Training needs to be matched with career progression opportunities for qualified clinical pharmacologists. All tertiary/academic hospitals should create posts for specialist clinical pharmacologists, who would play key roles in training healthcare professionals who prescribe medicines, provide patient care, conduct clinical research, and influence regional drug policy. In developing the national health insurance (NHI) scheme, ${ }^{[9]}$ clinical pharmacologists would be indispensable members of therapeutic teams in all relevant health facilities.

Finally: Decision-makers need to understand the many roles that clinical pharmacologists play. The WHO position paper on the roles of clinical pharmacologists in healthcare, teaching and research ${ }^{[2]}$ clearly lays out the benefits that clinical pharmacologists bring to the delivery of better healthcare.

1. Department of Health (Government notice no. R 645). The Health Professional Act 1974 (Act No. 56 of 1974): Regulations relating to the specialties and sub-specialties in medicine and dentistry: Amendment. 22 August 2012. Pretoria: DoH, 2012. http://www.greengazette.co.za (accessed 10 November 2012).

2. Rago L, Sjolin-Forsberg G, Orme M, Sjoqvist F, Birkett D, eds. Clinical Pharmacology in Health Care, Teaching and Research. WHO/CIOMS/IUPHAR, 2012. www.cioms.ch (accessed 10 November 2012). World Health Organization. The World Medicines Situation. Geneva: World Health Organization, 2004. http://www.searo.who.int/LinkFiles/Reports_World_Medicines_Situation.pdf (accessed 10 November 2012).

4. Smith AJ. Unfinished business: Clinical pharmacology and world health. Int J Risk Saf Med 2005;17:6571. http://apps.who.int/medicinedocs/pdf/s7918e/s7918e.pdf (accessed 10 November 2012).

Mayosi BM, Dhai A, Folb P, et al Consensus Report on Revitalising Clinical Research in South Africa. Pretoria: Academy of Science of South Africa, November 2009. http://www.assaf.co.za/wp-content/ Pretoria: Academy of Science of South Africa, November 2009. http://www.as
uploads/2009/09/ASSAf-Clinical-Report-2009.pdf (accessed 10 November 2012).

6. Siegfried N, Volmink J, Dhansay A. Does South Africa need a national clinical trials support unit? S Afr Med J 2010;100(8):521-524.

7. World Health Organization. Promoting Rational Use of Medicines: Core Components. WHO Policy Perspectives on Medicines, No. 5. Geneva: World Health Organization, 2002. http://apps. whoint/ medicinedocs/pdf/h3011e/h3011e.pdf (accessed 10 November 2012).

8. Holloway K, van Dijk L, eds. The World Medicines Situation 2011: Rational Use of Medicines. 3rd ed. Geneva: World Health Organization, 2011. http://apps. who.int/medicinedocs/documents/s18064en/ s18064en.pdf (accessed 10 November 2012).

9. Department of Health A Booklet - National Health Insurance: Healthcare for all South Africans. Pretoria DoH, 2012. http://www.doh.gov.za/docs/publicity/2011/nhi_english.pdf (accessed 10 November 2012).

Accepted 18 December 2012. 